\title{
Genetic Simulated Annealing Algorithm for QoS Routing
}

\author{
Zhang Hui \\ Information Engineering College \\ Zhongzhou University \\ Zhengzhou, China,450044 \\ huangjihai@sina.com
}

\author{
Huang Jihai \\ Information Engineering College \\ Zhongzhou University \\ Zhengzhou, China, 450044
}

\begin{abstract}
This paper proposes the math model of QoS routing. Then we present a genetic simulated annealing algorithm is proposed of QoS routing. The results show that the new method to enhance the search ratio and the speed of convergence have been markedly improved, the phenomenon of premature convergence was overcome.
\end{abstract}

Keywords-QoS routing, delay constraint, search ratio, genetic simulated annealing algorithm

\section{INTRODUCTION}

With the era of video communication coming which integrates voice, data and images, a lot of real-time multimedia services in computer network, such as streaming media, video-on-demand, network war games and distributed database, are increasing rapidly, leading to much more intensive bandwidth consumption and network congestion[12]. To some extent, Multicast technology can solve the problem of network bandwidth bottlenecks in the video communication process by using less network bandwidth and so being able to fundamentally reduce the load of network, and It has become an hot spot in the research field of network.

Routing is the key problem of the multicast technology: how to construct a multicast tree having the minimum cost from the source node to each of destination nodes. Towards the real-time characteristic in most of the multimedia services, this paper focuses on delay-constrained multicast routing based on low-cost shortest path tree algorithm [3].

The current approaches for multicast routing problem[4-6] include heuristic algorithms[7] and genetic algorithm[8]. In this thesis, the application of genetic algorithm in the QoS multicast problem is studied.The genetic algorithm is a global random optimization method, which has so many advantages,such as concurrent searching and colony optimize, and has been applied successfully in many fields of NP complete problems. Therefore, genetic algorithm provides us some new methods to solve the QoS multicast routing problems.

The simulated annealing/genetic algorithm is proposed for Delay-Contrained Multicast Routing problem.The proposed algorithm introduces simulated annealing to genetic operators so that it can enhance the ability of the global search and the convergence speed. The routing encoding method is used, so the genetic operators can be operated comparatively easily. And the algorithm analyse and solve the loop problem in course of operation. Simulations show the proposed algorithm can get low cost multicast tree without violating the delay constraint in fewer generations. With beter cost and convergence performance, the proposed algorithm can meet thedemands of the practical applications.

\section{MATHEMATICAL MODEL}

QoS multicast routing problems can be described as an undirected weighted graph $G=(V, E)$ [9-12], as shown in Figure 1. In the graph, vertices said the network nodes, edges said the network links, $\mathrm{V}$ is set of all the switching nodes in the network, E is set of all the edges in the network.

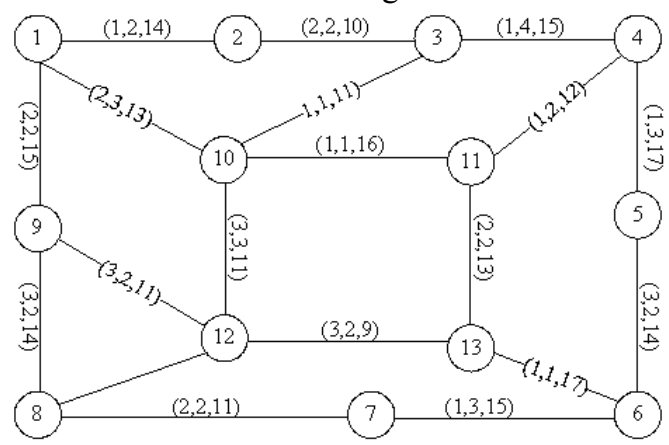

Figure 1. QoS routing topology

To facilitate the study, an assumption is made that there is at most only one link between a pair of nodes. In graph $\mathrm{G}$, any edge e corresponds to a triple $\left(c_{e}, b_{e}, d_{e}\right)$, where $c_{e}$ denotes the costs on the edge, $b_{e}$ denotes available bandwidth, and $d_{e}$ denotes the delay.

Let $R^{+}$the set of positive real numbers, For any link $e \in E$, the related QoS feature values were defined as:

(A) delay function Delay(e): $E \rightarrow R^{+}$;

(B) cost function $\operatorname{Cost}(\mathrm{e}): E \rightarrow R^{+}$;

(C) bandwidth function Bandwidth(e): $E \rightarrow R^{+}$;

Let $p(s, d)$ be one directed path from s to $\mathrm{d}$, there are the following relationships: 


$$
\begin{aligned}
& \operatorname{Delay}(p(s, d))=\sum_{e \in p(s, d)} \operatorname{Delay}(e) \leq D_{p} \\
& \operatorname{Cost}(p(s, d))=\sum_{e \in p(s, d)} \operatorname{Cost}(e) \geq B_{p}
\end{aligned}
$$$$
\operatorname{Bandwidth}(p(s, d))=\min \{\text { Bandwidth }(e) \text {, }
$$$$
e \in p(s, d)\}
$$

where $D_{p}$ denotes the delay constraint and $B_{p}$ denotes the bandwidth constraint.

\section{Genetic Simulated ANNEALING Algorithm}

\section{A. Encoding}

In this paper, we used path-based approach to construct the coding, the path between source node $\mathrm{s}$ and destination node $\mathrm{d}$ according to the network topology was served as the chromosome coding. It consists of a series of natural numbers, the first location of chromosome is always source node, the middle is the sequence of nodes through this feasible path, last is always the destination node. The code of chromosomes is also variable length because the path is a variable length.

\section{B. Generate Initail Population}

Firstly, in the algorithm, we remove the links that do not meet the requirements of QoS-bandwidth and get a new network topology.

This pretreatment can ensure that all the individuals generated can meet the minimum bandwidth requirements, reduce the search space of the algorithm, and accelerate the convergence speed of the algorithm.

In the new network topology, initial population is generated by the way combining the weighted depth-first search method and roulette method.

The algorithm is described as follows:

Step 1: For an initial population with size of S, each edge is given weight $\mathrm{S}$;

Step 2: Depth-first searching the nodes from the special one, which is generated using roulette wheel selection method in accordance with the weights of edges adjacent to the node;

Step 3: If there exists a path to meet the requirements of $p$ $(\mathrm{s}, \mathrm{d})$, the path is selected as a chromosome of the initial population; at the same time the weights of all edges decrease by 1 ;

Step 4: Repeat step 2 and step 3, until the number of chromosomes meeting the requirements of population size is found.

The target function of this problem is:

$$
F=\frac{\operatorname{Cost}(p(s, d))}{\psi\left(\operatorname{Del} a y(p(s, d))-D_{p}\right)}
$$

where $\psi(x)=\left\{\begin{array}{ll}1 & x \leq 0 \\ \lambda & x>0\end{array}\right.$ denotes penalty function of delay metric $(0<\lambda<1)$, which penalties the paths that violate QoS constraint(delay), and the value of $\lambda$ determines the degree of punishment.

Fitness function is defined as:

$$
f=1 / F
$$

\section{Crossover Operation}

In this algorithm, the crossover operation is different from traditional single point crossover,It randomly selects two chromosomes $C_{i}$ and $C_{j}$ from the population, and randomly selected genes $c_{i}$ and $c_{j}$ from the same public position of chromosomes $C_{i}$ and $C_{j}$, find the same point from the path which $c_{i}$ and $c_{j}$ represented as the crossover point. The pseudo codes are as follows:

Intput: O1,O2-The old chromosome before crossover;

Output: N1,N2-The new chromosome after crossover;

Function CrossoverOperation

begin

for $\mathrm{i}=1$ to $\mathrm{n}$

$\mathrm{r}=\mathrm{rand}()$;

if $(r>0.5)$

$\mathrm{N} 1[\mathrm{i}]=\mathrm{O} 1[\mathrm{i}]$;

$\mathrm{N} 2[\mathrm{i}]=\mathrm{O} 2[\mathrm{i}]$;

else

$\mathrm{O} 1[\mathrm{i}]=\mathrm{N} 1[\mathrm{i}]$

$\mathrm{O} 2[\mathrm{i}]=\mathrm{N} 2[\mathrm{i}]$; endif endfor

end

\section{Mutation Operations}

The specific mutation operation of the algorithm process is described as follows: it selects a chromosome $\mathrm{C}$ from the population at set mutation probability $\mathrm{Pm}$, randomly selects an integer $\mathrm{g}$ from the chromosome $\mathrm{C}$, finds its corresponding path, and then randomly selects a node in the path as a variation point. The pseudo codes are as follows:

Intput: O-The old chromosome before mutation;

Output: $\mathrm{N}$-The new chromosome after mutation;

Function MutationOperation begin

$i=O$. choserand () ;

$r=\operatorname{rand}()$; if $\left(r<P_{m}\right)$

$N_{l}=O \cdot \operatorname{addpath}(1,2,3, \ldots, i)$;

$N_{2}=O \cdot \operatorname{rand}(i+1, \ldots, m)$;

$N=N_{1}+N_{2}$; endif

end 


\section{E. Annealing Mutation Operator}

Annealing mutation operator is the key integration points of genetic algorithms and simulated annealing algorithm, annealing mutation operator specific description is as follows

Step1. Generate random numbers rand, if the mutation probability $\mathrm{Pm}>$ rand, then do the following, otherwise do nothing;

Step2. According to (5) calculated evaluation value f;

Step3. Randomly change a gene to get a new individual;

Step4. According to (5), getting a new evaluation value calculated $f\left(x^{\prime}\right)$, and calculating the difference between appraisal value $\triangle \mathrm{f}=\mathrm{f}\left(\mathrm{x}^{\prime}\right)-\mathrm{f}(\mathrm{x})$;

Step5. If $f\left(x^{\prime}\right)>f(x)$, it is explains that the new individual is better than the old individual, the new individual replaces the old individual; if $\mathrm{f}\left(\mathrm{x}^{\prime}\right) \leq \mathrm{f}(\mathrm{x})$, then the new individual is worse than the old individual, but still with probability exp $(\triangle \mathrm{f} / \mathrm{T})$ replaces the old individual.

\section{Simulation AND ANALYSIS}

\section{A. Experimental Parameters}

To study the performance of the hybrid genetic simulated annealing algorithm, two experimental results of practical problems are given. The environment of the experiment is MathLab7.0 simulation and the PC in the Pentium IV $2.8 \mathrm{G} / 512 \mathrm{M}$. In the genetic simulated annealing algorithm experiment, we set the maximum genetic algebra 50, crossover probability 0.85 , mutation probability 0.005 , penalty factor 1.2.If the initial temperature of annealing is land the temperature coefficient $\mathrm{k}$ is 0.95 .

\section{B. Experimental Data}

In this paper, Salama network topology generation algorithm is used and it is an improvement of Waxman topology generation algorithm.

Network topology generator parameters are as follows: Number of nodes is 50, the link delay and the costs distribute uniformly from $1 \mathrm{~ms}$ to $6 \mathrm{~ms}$, and from 10 to 180 , respectively.

\section{Experimental result}

\section{(a) Convergence}

Counting the number of the most optimal solution in each iteration of the algorithm, the relation between the cost of the optimal multicast cost tree and the number of iterations is shown in Figure 2.

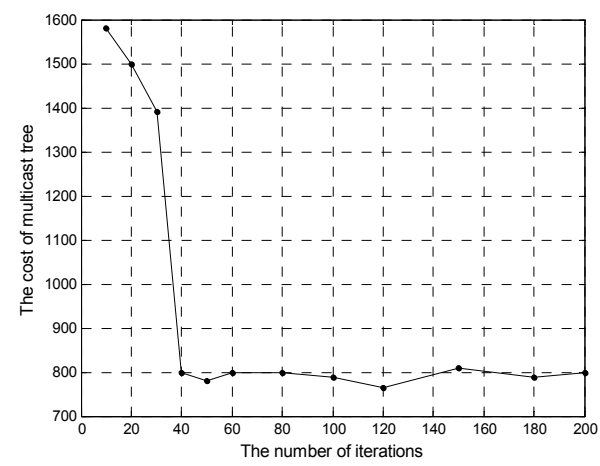

Figure 2. Relationships between number of iterations and cost of multicast tree

In Figure 2, the cost of the optimal multicast tree decreases quickly at the beginning of the algorithm; however, with the increase in the number of iterations, the cost does not decline any more after several generations in the evolution.

(b) Network cost performance

In order to study the performance of the network cost of this algorithm, we designed many simulations on different scale of network. We repeated for each network size of 20 experiments, and every time we take the average of the optimal multicast tree species as the scale of the optimal multicast network cost.

Fig. 3 is the relationships between the size of network and the optimal cost of multicast tree. The figure shows, the cost of the optimal multicast tree increases with the size of network.

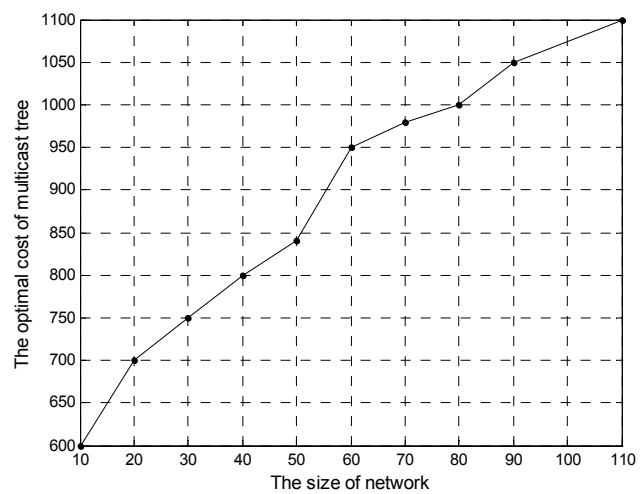

Figure 3. Relationships between size of network and the optimal cost of Multicast Tree

Fig.4 is the relationships between the number of destination node and the optimal cost of multicast tree. The figure shows, the cost of the optimal multicast tree increases with the number of destination node.

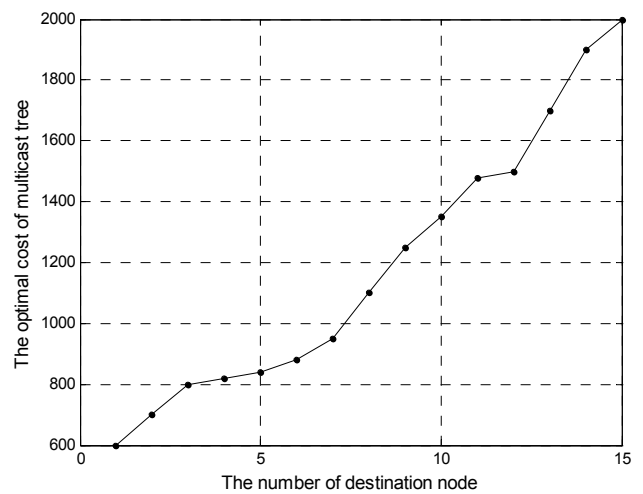

Figure 4. Relationships between number of destination node and the optimal cost of multicast tree 


\section{CONCLUSION}

This paper mainly studied the use of Qos routing. After a careful analysis of each binding condition in the Qos routing, a new mathematical model of Qos routing problem based on genetic simulated annealing algorithm is given. The simulation result proved that the algorithm in the thesis is correct and offered the proper solution to the problem in practice.

\section{REFERENCES}

[1] Huang Dong-jun. A Model and Its Realization of Multipoint Videoconferencing Based on Source-Specific Multicast (in Chinese) [J]. Chinese Journal of Electronics. 2005, 33(1):47-51.

[2] Zhang Miao. Survey on Application Layer Multicast (in Chinese)[J]. Chinese Journal of Electronics.2004,32(12a):22-23.

[3] Martin Zitterbart. Multicast Communication[M]. Morgan Kaufmann, 2001.

[4] Ruth Sara, Aguilar-Saven. Business process modeling: Review and framework $[\mathrm{J}]$. International Journal of Production Economics, 2004,90(2):129-149.
[5] DAML-S Coalition. DAML-S: Web service description for the semantic web[C]. Proc of 1st Int Semantic Web Conference, 2002:348-363.

[6] Aalst W M P.Formalization and verification of event-driven process chains[J]. Information and Software Technology,1999,41:639-650.

[7] YUAN Xin. Heuristic algorithms for multi-constrained quality-ofservice routing[J]. IEEE/ACM Transactions on Networking. 2002,10(2):244-256.

[8] Xiawei Z, Changjia C, Gang Z. A Genetic Algorithm for Multicasting Routing Problem. Proceedings of International Conference Communication Technology Proceedings, 2000, 1248-1253.

[9] Ruth Sara, Aguilar-Saven. Business process modeling: Review and framework $[\mathrm{J}]$. International Journal of Production Economics, 2004,90(20: 129-149.

[10] Jan Mendling, Carlo Simon. Business process design by view integration[C]. Vienna, Austria: Proceedings of Business Process Management Workshops,2006:55-64.

[11] Nirmit Desai,Munindar P Singh. Protocol-based business process modeling and enactment[C]. San Diego, CA, United States: Proceedings of IEEE International Conference on Web Services, 2004:35-42.

[12] Andrews T, Curbera F, Dholakia H,et al. Business process execution language for web services specification[R]. Version 1.1,2003. 